KEK Preprint 2002-107

\title{
Supersymmetric Quantum Mechanics with a Point Singularity
}

\author{
TAKASHI UCHINO* and IzUmi Tsutsui ${ }^{\dagger}$ \\ Institute of Particle and Nuclear Studies \\ High Energy Accelerator Research Organization (KEK) \\ Tsukuba 305-0801 \\ Japan
}

\begin{abstract}
We study the possibility of supersymmetry (SUSY) in quantum mechanics in one dimension under the presence of a point singularity. The system considered is the free particle on a line $\mathbb{R}$ or on the interval $[-l, l]$ where the point singularity lies at $x=0$. In one dimension, the singularity is known to admit a $U(2)$ family of different connection conditions which include as a special case the familiar one that arises under the Dirac delta $\delta(x)$-potential. Similarly, each of the walls at $x= \pm l$ admits a $U(1)$ family of boundary conditions including the Dirichlet and the Neumann boundary conditions. Under these general connection/boundary conditions, the system is shown to possess an $N=1$ or $N=2$ SUSY for various choices of the singularity and the walls, and the SUSY is found to be 'good' or 'broken' depending on the choices made. We use the supercharge which allows for a constant shift in the energy, and argue that if the system is supersymmetric then the supercharge is self-adjoint on states that respect the connection/boundary conditions specified by the singularity.
\end{abstract}

\footnotetext{
* email: uchino@post.kek.jp

$\dagger$ email: izumi.tsutsui@kek.jp
} 


\section{Introduction}

It has been known for some time that, in one dimension, quantum mechanics admits various different singular point interactions parametrized by the group $U(2)[1,2]$. These include the familiar singularity of the Dirac $\delta(x)$-potential of arbitrary strength which gives rise to discontinuity in the derivative of the wave function, but the generic connection condition in the $U(2)$ family develops discontinuity in both the wave function and its derivative. In mathematical terms, this is equivalent to the fact that the free Hamiltonian operator, defined on the line $\mathbb{R}$ with the singular point removed, admits a $U(2)$ family of self-adjoint extensions. If one considers an interval $[-l, l]$ with point singularity, then in addition to the $U(2)$ family of the singularity, the system is characterized further by the property of the endpoints $x= \pm l$ each of which has a $U(1)$ family of possible boundary conditions (see, e.g., $[1,3]$ ). These varieties in the connection/boundary conditions have been shown to accommodate interesting physical phenomena, such as duality, anholonomy (Berry phase) and scale anomaly, which are normally found in more complicated systems or in quantum field theory [4].

The varieties are also expected to furnish a room to realize novel quantum systems with supersymmetry (SUSY). In fact, in our previous work $[4,5]$, we found that there occurs a double degeneracy in the energy level for some specific choices of the conditions, and an attempt was made to reformulate the system into a SUSY quantum mechanics. There, we encountered the problem of how to ensure the self-adjointness of the supercharge under the given connection/boundary conditions. Another problem that needs to be addressed is how to preserve the conditions under the transformations generated by the supercharge. These properties are crucial for the very benefit of SUSY and should be maintained, since otherwise the generic degeneracy in the level and/or the positive semi-definiteness of the energy will not be guaranteed.

In this paper, we provide a full analysis on the possibility of SUSY quantum mechan-

ics for these systems, i.e., a free particle on the line $\mathbb{R}$ or on the interval $[-l, l]$ with a point singularity at $x=0$. We find that, for a large variety of the connection/boundary conditions, these systems indeed possess an $N=1$ or $N=2$ SUSY (the latter case being the Witten model [6]). The supercharge we use is a slightly extended version of the conventional one and allows for a constant shift in the energy. With this supercharge, the two properties mentioned above are shown to be maintained fully, if one takes the energy shift into account. The examples presented include cases where the SUSY is 'good' or 'broken' [7], showing that these systems, though being simple, embody the essential features of SUSY quantum mechanics observed in other models so far. 


\section{Supersymmetry on a line with point singularity}

Let us first explore the possibility of SUSY on a line $\mathbb{R}$ in the presence of a point singularity at $x=0$. The system is defined by the free Hamiltonian, $H=-\frac{\hbar^{2}}{2 m} \frac{d^{2}}{d x^{2}}$, on $\mathbb{R}$ with the point $x=0$ removed, and the singularity can be characterized by a set of connection conditions at $x=0$ for the wave function $\psi(x)$ belonging to the Hilbert space $\mathcal{H}=L^{2}(\mathbb{R} \backslash\{0\})$. The system may equally be formulated by cutting the space in half (see Fig.1) and identifying $\mathcal{H}$ with $L^{2}\left(\mathbb{R}^{+}\right) \otimes \mathbb{C}^{2}$, where instead of $\psi(x)$ one considers the vector-valued wave function,

$$
\Psi(x)=\left(\begin{array}{c}
\psi_{+}(x) \\
\psi_{-}(x)
\end{array}\right), \quad x \in \mathbb{R}^{+},
$$

defined from $\psi(x)$ by $\psi_{+}(x)=\psi(x)$ for $x>0$ and $\psi_{-}(-x)=\psi(x)$ for $x<0$. This way we introduce a $\mathbb{C}^{2}$-graded structure into the system allowing for accommodating SUSY, where now the Hamiltonian takes the form

$$
H=-\frac{\hbar^{2}}{2 m} \frac{d^{2}}{d x^{2}} \otimes I
$$

with $I$ being the identity matrix acting on the $\mathbb{C}^{2}$ vector.

Before proceeding further, let us recall that the set of connection conditions which ensures the self-adjointness of the Hamiltonian $H$ in (2.2) is provided by $[8,9,4]$

$$
(U-I) \Psi(+0)+i L_{0}(U+I) \Psi^{\prime}(+0)=0 .
$$

Here $U$, called the 'characteristic matrix', is an arbitrary $U(2)$ matrix characterizing uniquely the self-adjoint domain of $H$, which we denote by $\mathcal{D}_{U}(H)$, and we used $\Psi^{\prime}(x)=\frac{d}{d x} \Psi(x) .{ }^{1}$ The conditions (2.3) may also be written as

$$
U \Psi^{(+)}(+0)=\Psi^{(-)}(+0), \quad \text { with } \quad \Psi^{( \pm)}=\Psi \pm i L_{0} \Psi^{\prime} .
$$

A system is said to be 'supersymmetric' if it has self-adjoint operators $Q_{i}, i=1,2, \ldots$, called supercharges, such that $\left\{Q_{i}, Q_{j}\right\}=H \delta_{i j}$ (see, e.g., [7]). For the free Hamiltonian, the standard form of the supercharges is $Q_{i}=-i \lambda \frac{d}{d x} \otimes \sigma_{i}$ where $\lambda=\hbar / \sqrt{2 m}$ and $\sigma_{i}$ are the Pauli matrices. Formally, these supercharges satisfy the relation with the Hamiltonian $H$ in (2.2). However, this is not quite sufficient to prove that the system has a SUSY, since

\footnotetext{
1 We note that $\Psi^{\prime}$ used in [4] has an extra minus sign in the second component, but this sign factor is unnecessary here due to the mapping of the negative coordinate to the positive one.
} 


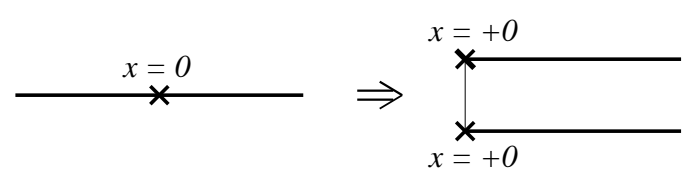

Figure 1. The system on a line $\mathbb{R}$ with a point singularity at $x=0$ may be identified with the system of two half lines $\mathbb{R}^{+}$with the probability flow between the two ends $x=0$ allowed.

operators are defined not just by the differential operations but also by the domains on which they operate. In fact, the supercharges $Q_{i}$ may not preserve the self-adjoint domain $\mathcal{D}_{U}(H)$ of the Hamiltonian for a given $U$. This can be seen, for example, by considering the domain $\mathcal{D}_{U}(H)$ for $U=\sigma_{3}$, for which the connection conditions read $\psi_{+}^{\prime}(+0)=\psi_{-}(+0)=$ 0 . The state $\Psi(x)=\left(0, x e^{-x}\right)^{T}$ belongs to $\mathcal{D}_{U}(H)$ but the transformed state $Q_{1} \Psi(x)=$ $\left(i \lambda(x-1) e^{-x}, 0\right)^{T}$ do not fulfill the connection conditions and hence $Q_{1} \Psi(x) \notin \mathcal{D}_{U}(H)$. This problem is generic for any domain $\mathcal{D}_{U}(H)$, because the supercharges $Q_{i}$ involve a derivative and, accordingly, they generate a state given basically by the derivative of the original state. Obviously, there is no reason to expect the generated state to remain in the same domain as the original. Under these circumstances, all we can hope for is perhaps to demand that the supercharges map any eigenstate of the Hamiltonian $H$ to some (but not necessarily the same) eigenstate of $H$ specified by the same $U$. This is reasonable because the benefit of SUSY in quantum mechanics is that it may lead to the degeneracy of energy levels by generating an eigenstate from a known eigenstate with the same energy by the operation of $Q_{i}$. If this is the property we want for SUSY in the system, then we do not need to require $Q_{i}$ to preserve the domain $\mathcal{D}_{U}(H)$, as long as the above demand for eigenstates is fulfilled.

To seek for supercharges fulfilling this demand, let us consider a slightly more general form than the standard one,

$$
Q=-i \lambda \frac{d}{d x} \otimes \sigma_{\vec{a}}+\mathbf{1} \otimes \sigma_{\vec{b}},
$$

where $\mathbf{1}$ is the identity operator in $L^{2}\left(\mathbb{R}^{+}\right)$and

$$
\sigma_{\vec{a}}=\sum_{i=1}^{3} a_{i} \sigma_{i}, \quad \sigma_{\vec{b}}=\sum_{i=1}^{3} b_{i} \sigma_{i}, \quad|\vec{a}|=1, \quad \vec{a} \cdot \vec{b}=0, \quad a_{i}, b_{i} \in \mathbb{R} .
$$

The standard form is obtained if we choose $\vec{a}=(1,0,0)$ or $(0,1,0)$ and $\vec{b}=0$. The properties (2.6) for the vectors $\vec{a}$ and $\vec{b}$ lead to the relation $2 Q^{2}=H+|\vec{b}|^{2}$. Now, given a 
domain $\mathcal{D}_{U}(H)$ of the Hamiltonian $H$ specified by the conditions (2.3) with some $U$, our demand for $Q$ to be a supercharge is that

$$
Q \Psi(x) \in \mathcal{D}_{U}(H)
$$

for any $\Psi(x)$ which is an eigenstate, $H \Psi(x)=E \Psi(x)$, of the Hamiltonian $H$. If there are two (or more) such independent operators, then one may choose an appropriate basis $Q_{i}$ whereby one has

$$
\left\{Q_{i}, Q_{j}\right\}=\left(H+|\vec{b}|^{2}\right) \delta_{i j}
$$

We note that our generalized supercharge (2.5) allows the constant shift $|\vec{b}|^{2}$ in (2.8) for $\vec{b} \neq 0$, which is not harmful to SUSY since the shift can always be absorbed into the Hamiltonian by the corresponding energy shift. It is, however, important to notice that due to the constant in the SUSY algebra (2.8) the Hamiltonian $H$ in (2.2) may no longer be positive semi-definite even in the presence of SUSY. Accordingly, the question of the system being good SUSY or broken SUSY [7] will be examined by taking this energy shift into account; they are seen by the property of whether the supercharges annihilate the ground state, $Q_{i} \Psi^{\text {grd }}=0$, rather than whether a zero energy state exists or not.

To examine if a given $U$ admits such supercharges, we first note that any $U \in U(2)$ can be decomposed as $U=V^{-1} D V$ with some matrix $V \in S U(2)$ and a diagonal matrix,

$$
D=\left(\begin{array}{cc}
e^{i \theta_{+}} & 0 \\
0 & e^{i \theta_{-}}
\end{array}\right), \quad \theta_{ \pm} \in[0,2 \pi)
$$

Observe that, in view of the conditions (2.3) which specify the self-adjoint domains corresponding to $U$, if $\Psi(x) \in \mathcal{D}_{U}(H)$ then $W \Psi(x) \in \mathcal{D}_{W U W^{-1}}(H)$ for any $W \in U(2)$. This implies that, if there exists a pair $(U, Q)$ satisfying the above demand, so does the pair $\left(W U W^{-1}, W Q W^{-1}\right)$ where $W Q W^{-1}$ is again written in the form (2.5). Choosing in particular $W=V$, we find that the pair $\left(D, V Q V^{-1}\right)$ also satisfies the demand. For this reason, with no loss of generality, we restrict ourselves below to the case where $U$ is diagonal. In other words, once a solution $(D, Q)$ is found for some $D$ and $Q$, then $\left(U, V^{-1} Q V\right)$ gives the desired solution.

To find the solutions $(D, Q)$, we observe that the charge $Q$ in $(2.5)$ induces the transformation on an eigenstate (with energy $E$ ) and its derivative as

$$
\begin{aligned}
\Psi(x) & \mapsto-i \lambda \sigma_{\vec{a}} \Psi^{\prime}(x)+\sigma_{\vec{b}} \Psi(x), \\
\Psi^{\prime}(x) & \mapsto-i \lambda \sigma_{\vec{a}} \Psi^{\prime \prime}(x)+\sigma_{\vec{b}} \Psi^{\prime}(x)=i \lambda^{-1} E \sigma_{\vec{a}} \Psi(x)+\sigma_{\vec{b}} \Psi^{\prime}(x)
\end{aligned}
$$


Our demand that the transformed state satisfy the same connection conditions (2.3), or (2.4), then implies

$$
\begin{aligned}
{\left[\left\{-\lambda(D-I) \sigma_{\vec{a}}+2 L_{0} D \sigma_{\vec{b}}\right\}\right.} & +\left\{\lambda(D-I) \sigma_{\vec{a}}-2 L_{0} \sigma_{\vec{b}}\right\} D \\
& \left.-L_{0}^{2} \lambda^{-1} E(D+I) \sigma_{\vec{a}}(D+I)\right] \Psi^{(+)}(+0)=0 .
\end{aligned}
$$

An important point to be noted is that the original conditions (2.4) provide relations among the components between the two vectors $\Psi^{(+)}(+0)$ and $\Psi^{(-)}(+0)$, but not among those within each of the vectors. This means that the equality (2.11) holds without the vector $\Psi^{(+)}(+0)$. We then see that, since the energy $E$ varies with the eigenstate considered, for the conditions (2.11) to be identical to the original ones (2.3) which are independent of $E$, the last term in the square bracket in (2.11) must vanish separately from the rest. The conditions for SUSY therefore become

$$
(D+I) \sigma_{\vec{a}}(D+I)=0
$$

and

$$
\lambda(D-I) \sigma_{\vec{a}}(D-I)+2 L_{0}\left[D, \sigma_{\vec{b}}\right]=0
$$

From (2.12) one obtains $\operatorname{det}(D+I)=0$, and from (2.13) one finds $D \neq-I$. This shows that one of the eigenvalues of $D$ must be -1 while the other cannot be -1 . Since the two eigenvalues in (2.9) can be interchanged by the conjugation $D \rightarrow W D W^{-1}$ with $W=i \sigma_{1}$, the diagonal matrix $D$ can always be taken to be

$$
D=\left(\begin{array}{cc}
e^{i \theta} & 0 \\
0 & -1
\end{array}\right), \quad \theta \neq \pi .
$$

For this $D$ the condition (2.12) is fulfilled if

$$
\sigma_{\vec{a}}=\cos \alpha \sigma_{1}+\sin \alpha \sigma_{2}=e^{-i \frac{\alpha}{2} \sigma_{3}} \sigma_{1} e^{i \frac{\alpha}{2} \sigma_{3}},
$$

where $\alpha \in[0,2 \pi)$ is an arbitrary angle parameter. If we consider in (2.5) the simple supercharge $Q$ with $\vec{b}=0$, then from (2.13) we have $\theta=0$, i.e., $D=\sigma_{3}$ and the supercharge $Q$ specified by the $\sigma_{\vec{a}}$ in (2.15). For $Q$ with $\vec{b} \neq 0$, we combine (2.12) and (2.13) to find

$$
\left[\sigma_{3}, \sigma_{\vec{b}}\right]=\frac{2 i \lambda}{L(\theta)} \sigma_{\vec{a}}
$$

where we have defined

$$
L(\theta)=L_{0} \cot \frac{\theta}{2}
$$


which provides a physical length scale to the system [10]. The condition (2.16) can then be solved by

$$
\sigma_{\vec{b}}=\frac{\lambda}{L(\theta)}\left\{\sin \alpha \sigma_{1}-\cos \alpha \sigma_{2}\right\}+c \sigma_{3}=-\frac{\lambda}{L(\theta)} e^{-i \frac{\alpha}{2} \sigma_{3}} \sigma_{2} e^{i \frac{\alpha}{2} \sigma_{3}}+c \sigma_{3},
$$

with $c \in \mathbb{R}$ being arbitrary. Collecting all, we find that the supercharge takes the form $Q=q(\alpha, c ; \theta)$ with

$$
q(\alpha, c ; \theta)=-i \lambda \frac{d}{d x} \otimes e^{-i \frac{\alpha}{2} \sigma_{3}} \sigma_{1} e^{i \frac{\alpha}{2} \sigma_{3}}+\mathbf{1} \otimes\left[-\frac{\lambda}{L(\theta)} e^{-i \frac{\alpha}{2} \sigma_{3}} \sigma_{2} e^{i \frac{\alpha}{2} \sigma_{3}}+c \sigma_{3}\right] .
$$

For the general $U=V^{-1} D V$, the supercharge is given by

$$
Q=V^{-1} q(\alpha, c ; \theta) V
$$

as noted above.

We therefore have learned that, if the characteristic matrix $U$ has eigenvalues -1 and $e^{i \theta} \neq-1$, the system admits two independent supercharges, i.e., an $N=2$ SUSY. For other $U$, no SUSY is allowed under the $Q$ in (2.5). Since the conjugation by $V$ in $(2.20)$ merely rotates the vectors in the basis $\sigma_{i}$, for any $U$ that enjoys the $N=2$ SUSY one may use the concise basis set of supercharges,

$$
Q_{1}=-i \lambda \frac{d}{d x} \otimes \sigma_{1}-\frac{\lambda}{L(\theta)} \otimes \sigma_{2}, \quad Q_{2}=-i \lambda \frac{d}{d x} \otimes \sigma_{2}+\frac{\lambda}{L(\theta)} \otimes \sigma_{1},
$$

by setting $\alpha=0$ or $\alpha=\pi / 2$ with $c=0$. These supercharges satisfy (2.8) with $|\vec{b}|^{2}=$ $[\lambda / L(\theta)]^{2}$. We remark that, if we restrict ourselves to the simple $Q$ with $\vec{b}=0$, then from (2.12) and (2.13) we find that an $N=2$ SUSY arises only if the eigenvalues of $U$ are \pm 1 .

\section{Supersymmetry on an interval with point singularity}

Next we investigate the possibility of SUSY in the system of an interval with a point singularity (i.e., a quantum well with a point interaction). Our assumption for the supercharges remains to be of the form (2.5), but now we need to take into account the boundary effect at both ends of the interval. Let the interval be $[-l, l]$ with the point singularity placed at $x=0$. As before, we remove the point $x=0$ from the interval and identify the Hilbert space $\mathcal{H}=L^{2}([-l, l] \backslash\{0\})$ with $L^{2}((0, l]) \otimes \mathbb{C}$. The Hamiltonian (2.2) then possesses self-adjoint domains $\mathcal{D}_{\tilde{U}}(H)$, where now the characteristic matrix $\tilde{U}$ belongs to $U(2) \times U(1) \times U(1)$ because the point singularity at $x=0$ furnishes a $U(2)$ arbitrariness 


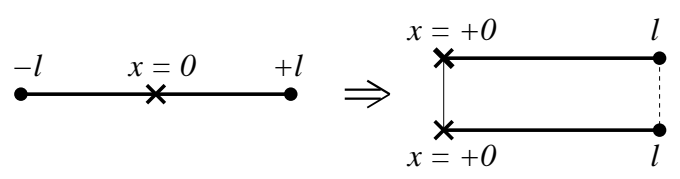

Figure 2. The system on the interval $[l,-l]$ with a point singularity at $x=0$ may be identified with the system of two half intervals $(0, l]$ with the probability flow between the two ends $x=0$ allowed. The flow is not allowed at the other two ends $x=l$.

while each $U(1)$ corresponds to the arbitrariness provided by the two ends at $x= \pm l$. We write $\tilde{U}=U \times D_{l}$ where $U \in U(2)$ is the characteristic matrix associated with $x=0$ and $D_{l} \in U(1) \times U(1)$ is the one associated with the two ends $x= \pm l$. If we regard the two ends as a special case of point singularity where no probability flow is allowed (see Fig.2), then in terms of the boundary vectors $\Psi(l)$ and $\Psi^{\prime}(l)$, the matrix $D_{l}$ may be taken to be a diagonal $U(1) \times U(1)$ matrix embedded in $U(2)$. With these, the self-adjoint domain $\mathcal{D}_{\tilde{U}}(H)$ can be specified by the connection conditions at $x=0$,

$$
(U-I) \Psi(+0)+i L_{0}(U+I) \Psi^{\prime}(+0)=0
$$

together with the boundary conditions at $x= \pm l$,

$$
\left(D_{l}-I\right) \Psi(l)+i L_{0}\left(D_{l}+I\right) \Psi^{\prime}(l)=0 .
$$

Our task is again to find a pair $\left(\tilde{U}=U \times D_{l}, Q\right)$ such that the supercharge $Q$ preserve the connection/boundary conditions (3.1) and (3.2). Recall that a supercharge that preserves the conditions (3.1) and (3.2) specified by $U$ and $D_{l}$, respectively, exists only if $U$ and $D_{l}$ are of the form (2.14) for $\theta \neq \pi$, up to the conjugation by some $V \in S U(2)$ for $U$. We therefore have

$$
U=V^{-1} D V, \quad D=\left(\begin{array}{cc}
e^{i \theta} & 0 \\
0 & -1
\end{array}\right), \quad D_{l}=\left(\begin{array}{cc}
e^{i \theta_{l}} & 0 \\
0 & -1
\end{array}\right)
$$

for $\theta, \theta_{l} \neq \pi$, and our question is whether there is a supercharge $Q$ compatible to both of the conditions (3.1) and (3.2). In terms of $q(\alpha, c ; \theta)$ in (2.19), the supercharge corresponding to $x=0$ is $V^{-1} q(\alpha, c ; \theta) V$ whereas the one corresponding to $x= \pm l$ is $q\left(\alpha_{l}, c_{l} ; \theta_{l}\right)$, and hence our requirement of compatibility reads

$$
Q=V^{-1} q(\alpha, c ; \theta) V=q\left(\alpha_{l}, c_{l} ; \theta_{l}\right)
$$


Comparing the terms involving the derivative in (3.4), one finds

$$
\operatorname{tr} \sigma_{i}\left[V^{-1} e^{-i \frac{\alpha}{2} \sigma_{3}} \sigma_{1} e^{i \frac{\alpha}{2} \sigma_{3}} V\right]=\operatorname{tr} \sigma_{i}\left[e^{-i \frac{\alpha_{l}}{2} \sigma_{3}} \sigma_{1} e^{i \frac{\alpha_{l}}{2} \sigma_{3}}\right]
$$

for $i=1,2,3$, which can be made more explicit by using the parametrization

$$
V=e^{i \frac{\mu}{2} \sigma_{2}} e^{i \frac{\nu}{2} \sigma_{3}}, \quad \mu \in[0, \pi], \quad \nu \in[0,2 \pi)
$$

as

$$
\begin{aligned}
\cos \alpha \cos \mu \cos \nu-\sin \alpha \sin \nu & =\cos \alpha_{l}, \\
\cos \alpha \cos \mu \sin \nu+\sin \alpha \cos \nu & =\sin \alpha_{l}, \\
\cos \alpha \sin \mu & =0 .
\end{aligned}
$$

These are satisfied if

$$
\mu=0, \quad \alpha_{l}=\nu+\alpha, \quad \text { or } \quad \mu=\pi, \quad \alpha_{l}=\nu-\alpha \pm \pi
$$

or otherwise if

$$
\alpha=\frac{\pi}{2}, \quad \alpha_{l}=\nu+\frac{\pi}{2}, \quad \text { or } \quad \alpha=\frac{3 \pi}{2}, \quad \alpha_{l}=\nu-\frac{\pi}{2} .
$$

The remaining conditions which arise from the non-derivative term in (3.4) are

$$
\begin{array}{r}
\operatorname{tr} \sigma_{i}\left[-\frac{\lambda}{L(\theta)} V^{-1} e^{-i \frac{\alpha}{2} \sigma_{3}} \sigma_{2} e^{i \frac{\alpha}{2} \sigma_{3}} V+c V^{-1} \sigma_{3} V\right] \\
=\operatorname{tr} \sigma_{i}\left[-\frac{\lambda}{L\left(\theta_{l}\right)} e^{-i \frac{\alpha_{l}}{2} \sigma_{3}} \sigma_{2} e^{i \frac{\alpha_{l}}{2} \sigma_{3}}+c_{l} \sigma_{3}\right] .
\end{array}
$$

Among these conditions the one corresponding to the third component $i=3$ can always be fulfilled by adjusting the free constants $c_{l}$ and $c$. Also, only one of the remaining two components, say $i=2$, is important because, once its corresponding condition is met, the construction of the supercharge ensures that the other component $i=1$ fulfills its condition, too. The $i=2$ component of $(3.10)$ is

$$
-\frac{\lambda}{L(\theta)}(\cos \alpha \cos \nu-\sin \alpha \cos \mu \sin \nu)+c \sin \mu \sin \nu=-\frac{\lambda}{L\left(\theta_{l}\right)} \cos \alpha_{l} .
$$

Now, for (3.8) we observe that (3.11) simplifies into $L(\theta)= \pm L\left(\theta_{l}\right)$ or $\theta= \pm \theta_{l}$. We thus realize that, if the point singularity at $x=0$ and the endpoints at $x= \pm l$ are characterized by

$$
U=\left(\begin{array}{cc}
e^{i \theta} & 0 \\
0 & -1
\end{array}\right), \quad D_{l}=\left(\begin{array}{cc}
e^{i \theta} & 0 \\
0 & -1
\end{array}\right), \quad \theta \neq \pi
$$


or

$$
U=\left(\begin{array}{cc}
-1 & 0 \\
0 & e^{-i \theta}
\end{array}\right), \quad D_{l}=\left(\begin{array}{cc}
e^{i \theta} & 0 \\
0 & -1
\end{array}\right), \quad \theta \neq \pi
$$

the system has the supercharge preserving the connection/boundary conditions simultaneously. The supercharge is

$$
Q=q(\alpha, c ; \theta)
$$

where $q(\alpha, c ; \theta)$ is given in (2.19). Since the angle parameter $\alpha$ in the derivative term in (3.14) remains arbitrary, we see that the system admits an $N=2$ SUSY.

On the other hand, for (3.9) we observe that (3.11) reduces to $\nu=0, \pi$, or

$$
\pm \frac{\lambda}{L(\theta)} \cos \mu+c \sin \mu= \pm \frac{\lambda}{L\left(\theta_{l}\right)}
$$

This relation may be used to determine the constant $c$ in favor of $\mu, \theta$ and $\theta_{l}$. The relevant supercharge then reads

$$
Q=q(\nu \pm \pi / 2, c ; \theta)
$$

where $\nu$ is one of the parameters in (3.6). In contrast to (3.14), the angle parameter in (3.16) is determined by $\nu$ in $U$, and hence the system admits only an $N=1$ SUSY.

To see in more detail the content of the SUSY systems we have found, we consider, for instance, the case (3.12) whose connection/boundary conditions are

$$
\begin{array}{cc}
\psi_{+}(+0)+L(\theta) \psi_{+}^{\prime}(+0)=0, & \psi_{-}(+0)=0, \\
\psi_{+}(l)+L(\theta) \psi_{+}^{\prime}(l)=0, & \psi_{-}(l)=0 .
\end{array}
$$

The eigenstates of the Hamiltonian $H$ with positive energy $E_{n}=\hbar^{2} k_{n}^{2} /(2 m)>0$ satisfying (3.17) are doubly degenerate,

$$
\Psi_{+}^{(n)}(x)=N_{+}^{(n)}\left(\begin{array}{c}
-L(\theta) k_{n} \cos k_{n} x+\sin k_{n} x \\
0
\end{array}\right), \quad \Psi_{-}^{(n)}(x)=N_{-}^{(n)}\left(\begin{array}{c}
0 \\
\sin k_{n} x
\end{array}\right)
$$

where $k_{n}=n \pi / l$ with $n=1,2,3, \ldots$, and $N_{+}^{(n)}$ and $N_{-}^{(n)}$ are normalization constants. Under the SUSY transformations generated by (2.21), these two states interchange each other, $\Psi_{ \pm}^{(n)} \mapsto Q_{i} \Psi_{ \pm}^{(n)} \propto \Psi_{\mp}^{(n)}$ for $i=1,2$. The ground state is given by

$$
\Psi_{+}^{\operatorname{grd}}(x)=N_{+}^{\operatorname{grd}}\left(\begin{array}{c}
e^{-x / L(\theta)} \\
0
\end{array}\right)
$$

with energy $E^{\text {grd }}=-\hbar^{2} /\left\{2 m(L(\theta))^{2}\right\}=-[\lambda / L(\theta)]^{2}<0$, which exists for any $\theta \neq \pi$ except when $\theta=0$ which yields the Neumann condition, $\psi_{+}^{\prime}(+0)=\psi_{+}^{\prime}(l)=0$. The 
ground state is unique and annihilated by the supercharge $Q_{i} \Psi_{+}^{\text {grd }}=0$, which shows that the system possesses a good SUSY.

For the case (3.13), on the other hand, the connection/boundary conditions read

$$
\begin{array}{rlrl}
\psi_{+}(+0) & =0, & \psi_{-}(+0)-L(\theta) \psi_{-}^{\prime}(+0)=0, \\
\psi_{+}(l)+L(\theta) \psi_{+}^{\prime}(l) & =0, \quad \psi_{-}(l)=0 .
\end{array}
$$

The eigenstates with positive energy are then

$$
\Psi_{+}^{(n)}(x)=N_{+}^{(n)}\left(\begin{array}{c}
\sin k_{n} x \\
0
\end{array}\right), \quad \Psi_{-}^{(n)}(x)=N_{-}^{(n)}\left(\begin{array}{c}
0 \\
\sin k_{n}(x-l)
\end{array}\right)
$$

where the discrete $k_{n}>0$ are determined as solutions of $L(\theta) k_{n}+\tan \left(k_{n} l\right)=0$. Similarly, there arise the ground states,

$$
\Psi_{+}^{\mathrm{grd}}(x)=N_{+}^{\mathrm{grd}}\left(\begin{array}{c}
\sinh \kappa x \\
0
\end{array}\right), \quad \Psi_{-}^{\mathrm{grd}}(x)=N_{-}^{\mathrm{grd}}\left(\begin{array}{c}
0 \\
\sinh \kappa(x-l)
\end{array}\right)
$$

with $E^{\text {grd }}=-\hbar^{2} \kappa^{2} /(2 m)<0$, where $\kappa>0$ satisfies $L(\theta) \kappa+\tanh \kappa l=0$ which has a solution for $-l<L(\theta)<0$. If $L(\theta)=-l$, we have in addition the zero energy states,

$$
\Psi_{+}^{\text {zero }}(x)=N_{+}^{\text {zero }}\left(\begin{array}{l}
x \\
0
\end{array}\right), \quad \Psi_{-}^{\text {zero }}(x)=N_{-}^{\text {zero }}\left(\begin{array}{c}
0 \\
x-l
\end{array}\right)
$$

Irrespective of the energy (positive, negative or zero), all states are doubly degenerate and the degenerate pair of states are related by the SUSY transformations generated by $Q_{i}$. The degeneracy of the ground states implies that, in contrast to the previous case, the SUSY is broken here. Note that the energy of the ground states does not attain the lower bound, $E^{\text {grd }}>-[\lambda / L(\theta)]^{2}$.

In particular, if we choose $\theta=0$, then the conditions (3.17) become the Neumann type $\psi_{+}^{\prime}(+0)=0=\psi_{+}^{\prime}(l)$ and the Dirichlet type $\psi_{-}(+0)=0=\psi_{-}(l)$, whereas the conditions (3.20) become their combinations, $\psi_{+}(+0)=0=\psi_{+}^{\prime}(l)$ and $\psi_{-}^{\prime}(+0)=0=\psi_{-}(l)$. These are the models known earlier [7] whose supercharges are given without the constant term in (2.5). Taking into account the $N=1$ systems realized on the interval under (3.16), we see that, under the supercharge (2.5) with a constant term, the general point singularity leads to a much richer variety of SUSY systems than known before.

If we restrict ourselves to the simple $Q$ with $\vec{b}=0$, then from the result in section 2 , we know that both of $U$ and $D_{l}$ must have the eigenvalues +1 and -1 , that is, $U=V^{-1} \sigma_{3} V$ 


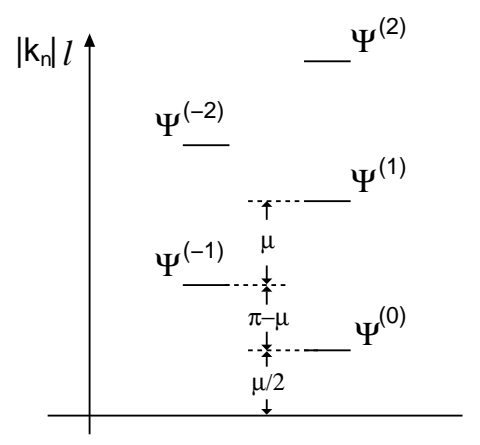

Figure 3. Energy levels of the $N=2$ SUSY system possessing the simple supercharge $Q$ with $\vec{b}=0$. The levels are not degenerate unless $\mu=0$ or $\pi$.

and $D_{l}=\sigma_{3}$ (up to the exchange of the eigenvalues). This implies the connection/boundary conditions,

$$
\begin{array}{rlrl}
e^{i \nu} \psi_{+}(+0)-\cot \frac{\mu}{2} \psi_{-}(+0) & =0, & e^{i \nu} \psi_{+}^{\prime}(+0)+\tan \frac{\mu}{2} \psi_{-}^{\prime}(+0)=0, \\
\psi_{+}^{\prime}(l) & =0, \quad \psi_{-}(l)=0,
\end{array}
$$

under which we have the eigenstates,

$$
\Psi^{(n)}(x)=N^{(n)}\left(\begin{array}{c}
-e^{-i \nu} \cos k_{n}(x-l) \\
\sin k_{n}(x-l)
\end{array}\right), \quad k_{n}=\frac{n \pi+\mu / 2}{l},
$$

for $n \in \mathbb{Z}$. Each eigenstate is invariant under the SUSY transformation by $Q$, and as shown in Fig.3, the energy levels $E^{(n)}=\hbar^{2} k_{n}^{2} /(2 m)$ are not degenerate unless $\mu=0$ or $\pi$.

For the interval we have yet another extension based on the 'half parity' transformation,

$$
\mathcal{X}: \Psi(x) \mapsto(\mathcal{X} \Psi)(x)=\left(\begin{array}{c}
\psi_{+}(l-x) \\
\psi_{-}(x)
\end{array}\right)
$$

which is well-defined in $\mathcal{H}$ and fulfills $\mathcal{X}^{2}=\mathrm{id}$. For the system characterized by $\tilde{U}=U \times D_{l}$ with $U$ and $D_{l}$ given either by (3.12) or (3.13), this half parity induces a change in the characteristic matrix $\tilde{U} \mapsto \tilde{U}^{\mathcal{X}}=U^{\mathcal{X}} \times D_{l}^{\mathcal{X}}$, namely, if $\Psi \in \mathcal{D}_{\tilde{U}}(H)$ then $\mathcal{X} \Psi \in \mathcal{D}_{\tilde{U}^{\mathcal{X}}}(H)$, where $U^{\mathcal{X}}$ and $D_{l}^{\mathcal{X}}$ are given by interchanging the upper left components of $U$ and $D_{l}$ with the extra sign $\theta \rightarrow-\theta$. Clearly, we have $Q(\mathcal{X} \Psi) \in \mathcal{D}_{\tilde{U}^{\mathcal{X}}}(H)$ for the supercharge $Q=\mathcal{X}_{q}(\alpha, c ; \theta) \mathcal{X}^{-1}$. The half parity transformation $\mathcal{X}$ leaves the good SUSY system (3.12) unaltered, but it turns the broken SUSY system (3.13) into one with

$$
U^{\mathcal{X}}=\left(\begin{array}{cc}
e^{-i \theta} & 0 \\
0 & e^{-i \theta}
\end{array}\right), \quad D_{l}^{\mathcal{X}}=\left(\begin{array}{cc}
-1 & 0 \\
0 & -1
\end{array}\right), \quad \theta \neq \pi
$$


Under these the connection/boundary conditions read

$$
\begin{aligned}
\psi_{+}(+0)-L(\theta) \psi_{+}^{\prime}(+0) & =0, & & \psi_{-}(+0)-L(\theta) \psi_{-}^{\prime}(+0)=0, \\
\psi_{+}(l) & =0, & & \psi_{-}(l)=0 .
\end{aligned}
$$

Evidently, this system has a spectrum identical to the case (3.13) and the $N=2$ SUSY is broken. The present case corresponds to the 'self-dual' subfamily mentioned earlier in Ref.[5], which pointed out that the system becomes a Witten model at $\theta=\pi$ but fell short of obtaining the full realization of SUSY quantum mechanics for other $\theta$ due to the question of the self-adjointness of the supercharge, which we now address next.

\section{Self-Adjointness of the supercharge}

An important question which remains to be answered is whether our supercharge $Q$ in (2.20) can be defined as a self-adjoint operator, and if so, whether its self-adjoint domain is compatible with the self-adjoint domain of the Hamiltonian.

To answer the former half of the question, we first observe that the problem of the self-adjointness can be examined by the form (2.5) because, analogously to the case of the Hamiltonian, a self-adjoint domain for (2.20), if any, can be obtained from a domain for (2.5) by the conjugation of the matrix characterizing the domain of the supercharge. Now, for the interval $[-l, l]$, for example, the supercharge (2.5) being self-adjoint implies

$$
\int_{+0}^{l} \Psi^{\dagger}(x)(Q \Psi)(x) d x-\int_{+0}^{l}(Q \Psi)^{\dagger}(x) \Psi(x) d x=0 .
$$

Clearly, the constant term in $Q$ drops out from this condition and hence does not affect the domain determined from (4.1). Taking the freedom of conjugation (including the half parity $\mathcal{X}$, if necessary) into account, with no loss of generality we can restrict ourselves to the simple form $Q=-i \lambda \frac{d}{d x} \otimes \sigma_{2}$. Then, the condition (4.1) reduces to

$$
\Psi^{\dagger}(l) \sigma_{2} \Psi(l)-\Psi^{\dagger}(+0) \sigma_{2} \Psi(+0)=0 .
$$

This can be fulfilled if $\Psi(0)=M \Psi(l)$ with some $U(1,1)$ matrix $M$, which satisfies $M^{\dagger} \sigma_{2} M=\sigma_{2}$. Other solutions are given by $\psi_{+}(+0)+u \psi_{-}(+0)=0$ and $\psi_{+}(l)+u_{l} \psi_{-}(l)=$ 0 , where $u, u_{l} \in \mathbb{R} \cup\{\infty\} \simeq U(1)$. The entire family of the solutions is given by the sum of these, and they actually form a $U(2)$ group. That this is the case may be argued by invoking the theory of self-adjoint extensions [1] as follows. 
Let $\mathcal{D}(Q)$ be a symmetric domain of $Q$ defined by

$$
\mathcal{D}(Q)=\left\{\Psi \mid \Psi \in A C([0, l]) \otimes \mathbb{C}^{2}, Q \Psi \in L^{2}((0, l]) \otimes \mathbb{C}^{2}, \Psi(+0)=\Psi(l)=0\right\},
$$

where $A C([0, l])$ denotes the space of absolutely continuous functions on the interval $[0, l]$. The domain of the adjoint $Q^{\dagger}$ is then given by

$$
\mathcal{D}\left(Q^{\dagger}\right)=\left\{\Psi \mid \Psi \in A C([0, l]) \otimes \mathbb{C}^{2}, Q \Psi \in L^{2}((0, l]) \otimes \mathbb{C}^{2}\right\} .
$$

The eigenvalue equation, $Q \Psi_{ \pm i}=\left(-i \lambda \frac{d}{d x} \otimes \sigma_{2}\right) \Psi_{ \pm i}= \pm i g \Psi_{ \pm i}$ for any $g>0$, has the solutions,

$$
\Psi_{ \pm i}^{(1)}=\left(\begin{array}{c} 
\pm i \\
1
\end{array}\right) e^{g x / \lambda}, \quad \Psi_{ \pm i}^{(2)}=\left(\begin{array}{c} 
\pm i \\
-1
\end{array}\right) e^{-g x / \lambda} .
$$

Thus the deficiency indices of the operator $Q$ are $(2,2)$ showing that the supercharge $Q$ admits a $U(2)$ family of self-adjoint extensions for the interval. These extensions are characterized by the aforementioned boundary conditions.

For the line $\mathbb{R}$, the above discussion applies more or less unchanged, except that the contribution from the endpoint $x=l$ is now absent. The deficiency indices of the operator $Q$ become $(1,1)$ since $\Psi_{ \pm i}^{(1)}$ are non-normalizable there. The resultant $U(1)$ family of selfadjoint domains is realized by the boundary condition, $\psi_{+}(+0)+u \psi_{-}(+0)=0$.

We next turn to the latter half of the question, namely, the compatibility of the two self-adjoint domains of $Q$ and $H$. This, again, can be answered affirmatively. In fact, we show that any self-adjoint domain $\mathcal{D}_{U}(H)$ is a subset of the self-adjoint domain of the supercharge $Q$ associated with the Hamiltonian $H$. To see this, let $f_{k}, k=1,2$ be the eigenvectors of the characteristic matrix $U$ specifying the Hamiltonian. We then have $U f_{k}=e^{i \theta_{i}} f_{k}$, where one of the eigenvalues, say $e^{i \theta_{2}}$, is -1 while the other $e^{i \theta_{1}}$ is not -1 . We now decompose the boundary vectors $\Psi(+0)$ and $\Psi^{\prime}(+0)$ into the eigenvectors of the characteristic matrix $U$ of the Hamiltonian,

$$
\Psi(+0)=\sum_{k=1}^{2}\left\langle f_{k}, \Psi(+0)\right\rangle f_{k}, \quad \Psi^{\prime}(+0)=\sum_{k=1}^{2}\left\langle f_{k}, \Psi^{\prime}(+0)\right\rangle f_{k},
$$

where $\langle\cdot, \cdot\rangle$ is the innerproduct for $\mathbb{C}^{2}$-vectors. In terms of these, the connection conditions (2.3) become

$$
\sum_{k=1}^{2}\left[\left(e^{i \theta_{i}}-1\right)\left\langle f_{k}, \Psi(+0)\right\rangle+i L_{0}\left(e^{i \theta_{i}}+1\right)\left\langle f_{k}, \Psi^{\prime}(+0)\right\rangle\right] f_{k}=0 .
$$


From the independence of the eigenvectors $f_{k}$ and $e^{i \theta_{2}}=-1$, we find

$$
\left\langle f_{2}, \Psi(+0)\right\rangle=0
$$

On the other hand, we recall that the existence of the supercharge requires (2.12). For $\sigma_{\vec{n}}$ now taken by $\sigma_{2}$, and for general $U,(2.12)$ becomes $(U+I) \sigma_{2}(U+I)=0$. Multiplying this by $f_{1}$ from the right and by $f_{1}^{\dagger} U^{\dagger}$ from the left, we obtain

$$
f_{1}^{\dagger} \sigma_{2} f_{1}=0
$$

From (4.8) and (4.9), we see that

$$
\Psi^{\dagger}(+0) \sigma_{2} \Psi(+0)=\sum_{j, k=1}^{2}\left\langle f_{k}, \Psi(+0)\right\rangle\left\langle\Psi(+0), f_{j}\right\rangle f_{j}^{\dagger} \sigma_{2} f_{k}=0 .
$$

So far we have considered only for $x=+0$, but the contribution from $x=l$ can be evaluated analogously to show that $\Psi^{\dagger}(l) \sigma_{2} \Psi(l)=0$. We therefore realize that the requirement for the self-adjointness (4.2) of $Q$ is ensured for any states belonging to the domain of $H$ for which an associated supercharge $Q$ exists, and that this is true for both the line and the interval system.

An important consequence of this is that all eigenvalues of $Q$ are real and, hence, the operator $Q^{2}$ is positive semi-definite. From this we find the lower bound of the spectrum, $H=2 Q^{2}-[\lambda / L(\theta)]^{2} \otimes \mathbf{1} \geq-[\lambda / L(\theta)]^{2} \otimes \mathbf{1}$, which is attained by the ground state (3.19) in the good SUSY case.

\section{Conclusion and discussions}

We have seen that a rich variety of $N=1$ and $N=2$ SUSY systems appear on the line and the interval with a singular point. The key element for this is that we consider the entire family of quantum singularities, and that we extend the supercharge by introducing a constant term allowing for a shift in the energy. The resultant $N=2$ SUSY systems are the Witten models and exhibit different features depending on the choice of the characteristic matrix that specifies the singularity.

The self-adjoint domain of the supercharge $Q$ is seen to contain the self-adjoint domain of the Hamiltonian $H$. As a result, the supercharge $Q$ may be expected to ensure the double degeneracy of energy levels by its operation on the eigenstates. Indeed, this has been seen in the first two (and the fourth) examples discussed in the interval case in section 3, but 
not in the third one where no degeneracy arises in general. The standard tool to establish the degeneracy is the Witten parity operator $W$, which is self-adjoint and satisfies $W^{2}=\mathbf{1}$, $[W, H]=0$ and $\{W, Q\}=0$. Obviously, for these conditions we need to examine if the domains of the operators invloved - in particular the domain $\mathcal{D}_{\tilde{U}}(H)$ of the Hamiltonian - change under the operation of $W$, and this is a highly nontrivial matter. However, if we assume that $W$ is given by a $2 \times 2$ Hermitian matrix acting on the graded Hilbert space, then on account of the boundary conditions $(2.3)$ we see that $\mathcal{D}_{\tilde{U}}(H)$ is preserved under $W$ if $[W, U]=\left[W, D_{l}\right]=0$. The first two (and the fourth) examples have their $\tilde{U}$ that fulfills this demand with $W=\sigma_{3}$, and consequently allow the degeneracy to occur in the eigenspaces of $\sigma_{3}$, i.e., the upper and the lower components of the vector states $\Psi$. In contrast, in the third example, $U$ and $D_{l}$ do not in general allow a common operator commuting the both simultaneously, implying that such $W$ cannot exist. In fact, this seems to be the case for a generic pair of $U$ and $D_{l}$ unless there underlies some mechanism to ensure the degeneracy.

Finally, we mention that it is straightforward to extend our analysis to more complicated systems with point singularities, including a circle with singular points or a quantum circuit whose vertices may be regarded as point singularities. For these systems, all we need is to put an appropriate $U(2)$ matrix to each of the point singularity and seek the supercharge that preserves the domain of the Hamiltonian. The extension may also include systems with a potential $V(x)$ which develops a singularity at its divergent point, such as the Coulomb potential. Singular potentials that arise in integrable models, such as the Calogero-Moser models, may also be of interest for the possibility of accommodating SUSY under the general singularity.

Acknowledgement: I.T. thanks T. Cheon and T. Fülöp for useful discussions. This work has been supported in part by the Grant-in-Aid for Scientific Research on Priority Areas (No. 13135206) by the Japanese Ministry of Education, Science, Sports and Culture. 


\section{References}

[1] M. Reed, B. Simon, "Methods of Modern Mathematical Physics", Vol.II, Academic Press, New York, 1980.

[2] S. Albeverio, F. Gesztesy, R. Høegh-Krohn and H. Holden, "Solvable Models in Quantum Mechanics", Springer, New York, 1988.

[3] T. Fülöp, T. Cheon and I. Tsutsui, Classical Aspects of Quantum Walls in One Dimension, KEK preprint 2001-134, quant-ph/0111057, to appear in Phys. Rev. A.

[4] T. Cheon, T. Fülöp and I. Tsutsui, Ann. Phys. 294 (2001) 1-23.

[5] I. Tsutsui, T. Fülöp and T. Cheon, J. Phys. Soc. Jpn. 69 (2000) 3473.

[6] E. Witten, Nucl.Phys. B 185 (1981) 513.

[7] G. Junker, "Supersymmetric Methods in Quantum and Statistical Physics", Springer, Berlin, 1996.

[8] N.I. Akhiezer and I.M. Glazman, "Theory of Linear Operators in Hilbert Space", Vol.II, Pitman Advanced Publishing Program, Boston, 1981.

[9] T. Fülöp and I. Tsutsui, Phys. Lett. 264A (2000) 366.

[10] I. Tsutsui, T. Fülöp and T. Cheon, Journ. Math. Phys. 42 (2001) 5687. 